\title{
Fiber Tract-Oriented Statistics for Quantitative Diffusion Tensor MRI Analysis ${ }^{\star}$
}

\author{
Isabelle Corouge $^{1,2}, \mathrm{P}$. Thomas Fletcher ${ }^{3}$, Sarang Joshi ${ }^{1,4}$, \\ John H. Gilmore ${ }^{2}$, and Guido Gerig ${ }^{1,2}$ \\ ${ }^{1}$ Departments of Computer Science, ${ }^{2}$ Psychiatry, ${ }^{4}$ Radiation Oncology, \\ University of North Carolina, Chapel Hill, USA \\ ${ }^{3}$ Scientific Computing and Imaging Institute, University of Utah, USA \\ corouge@unc.edu, fletcher@sci.utah.edu, sjoshi@unc.edu, \\ John_Gilmore@med.unc.edu, gerig@cs.unc.edu
}

\begin{abstract}
Diffusion tensor imaging (DTI) has become the major modality to study properties of white matter and the geometry of fiber tracts of the human brain. Clinical studies mostly focus on regional statistics of fractional anisotropy (FA) and mean diffusivity (MD) derived from tensors. Existing analysis techniques do not sufficiently take into account that the measurements are tensors, and thus require proper interpolation and statistics based on tensors, and that regions of interest are fiber tracts with complex spatial geometry. We propose a new framework for quantitative tract-oriented DTI analysis that includes tensor interpolation and averaging, using nonlinear Riemannian symmetric space. As a result, tracts of interest are represented by the geometry of the medial spine attributed with tensor statistics calculated within cross-sections. Examples from a clinical neuroimaging study of the early developing brain illustrate the potential of this new method to assess white matter fiber maturation and integrity.
\end{abstract}

Keywords: Diffusion tensor interpolation, diffusion tensor statistics, DTI analysis, fiber tract modeling.

\section{Introduction}

Diffusion tensor imaging of brain structures measures diffusion properties by the local probability of self-motion of water molecules. A tensor field is calculated from directional gradient images and characterizes amount and locally preferred directions of local diffusivity. While diffusion can be considered isotropic in fluid it appears highly anisotropic along neural fiber tracts due to inhibition of free diffusion of intra- and extracellular fluid. DTI has become the preferred modality to explore white matter properties

\footnotetext{
* This research is supported by the NIH NIBIB grant P01 EB002779, the NIMH Silvio Conte Center for Neuroscience of Mental Disorders MH064065, and the UNC Neurodevelopmental Disorders Research Center HD 03110. The work is also funded by the National Institutes of Health through the NIH Roadmap for Medical Research, Grant U54 EB005149-01, project NAMIC. We acknowledge the Insight Toolkit community for providing the software framework. Dr. Weili Lin, UNC Radiology, is acknowledged for active support of developing an improved neonatal DT MRI acquisition technique.
} 
associated with brain connectivity in vivo. The literature proposes a variety of DTI processing techniques, ranging from tensor field computation to quantitative analysis, and including visualization, regularization, registration, tractography and population statistics. Few of these methods make use of the full tensor information though most would benefit from an appropriate mathematical framework for tensor operations and tensor statistics calculation. For instance, tensor interpolation is required in regularization, registration and spatial normalization. Tensor statistics calculation becomes necessary for statistical DTI analysis in population studies. So far, analysis schemes have mostly focused on measuring properties in regions of interest and to a lesser extent along fiber bundles [1], [2], and they have not made use of the full tensor information. Consequently, clinical studies have mostly been limited to statistics of FA or MD maps on a voxel-by-voxel basis [3].

As opposed to voxel-based analysis, we propose an object-oriented approach in which the fiber tracts act as coordinate systems for quantitative DTI analysis. Our concept provides a complete representation of each individual bundle, describing both geometry and diffusion properties. The representation includes model of the geometry of individual bundles and statistics of diffusion tensors to be associated with the geometric model. In this paper, we focus on the computation of DTI tensor interpolation and DTI tensor statistics. Tensor information is integrated across cross-sections and represented along bundles. We thus supplement our previous model of tract geometry [4] with statistics of diffusion tensors, from which we derive diffusion properties.

\section{Theoretical Framework}

We denote the space of all diffusion tensors, i.e., the space of all $3 \times 3$ symmetric, positive-definite matrices, as $P D(3)$. Averaging and interpolation of diffusion tensors can be formulated as a least-squares minimization problem in this space. This definition depends on the choice of metric, or distance, on the space $P D(3)$. Treating diffusion tensors as vectors in the space $\mathbb{R}^{9}$, one can define a linear average of $N$ diffusion tensors $p_{1}, \ldots, p_{N}$ as $\mu=\frac{1}{N} \sum_{i=1}^{N} p_{i}$. This definition minimizes the Euclidean metric on $\mathbb{R}^{9}$. However, linear averages suffer from a "swelling" effect where diffusion tensors with the same determinant will have an average with a larger determinant. Linear interpolation of diffusion tensors suffers from this same effect. We adopt a more natural metric for averaging and interpolation by treating $P D(3)$ as a curved manifold, or more specifically, a Riemannian symmetric space. We use tensor averaging and interpolation methods, first presented in [5], [6], that are based on the notion of geodesic distance within this space. In a similar approach [7], interpolation is limited to only two tensors, whereas our work applies to averaging and interpolation of an arbitrary number of tensors and thus allows a full 3D interpolation of DTI data. The symmetric space metric does not suffer from the swelling effect of the linear metric, that is, diffusion tensors with the same determinant will have an average with the same determinant.

Symmetric spaces [8] arise from transformation groups on manifolds. The Riemannian metric is chosen to be invariant under the group transformations. The symmetric space structure of $P D(3)$ arises from transformations by $G L(3)$, the group of positivedeterminant matrices. The transformation of a diffusion tensor $p \in P D(3)$ by a matrix 
$g \in G L^{+}(3)$ is given by $p \mapsto g p g^{T}$. Because of the algebraic nature of the symmetric space structure, distance and geodesic computations on $P D(3)$ are also algebraic in nature. For instance, the geodesic distance between two tensors $p_{1}, p_{2} \in P D(3)$ can be computed using singular-value decomposition (SVD) as follows:

- Let $p_{1}=U \Lambda U^{T}$ be the SVD of $p_{1}$, set $g=U \sqrt{\Lambda}$.

- Compute the action of $g^{-1}$ on $p_{2}: y=g^{-1} p_{2}\left(g^{-1}\right)^{T}$.

- Again using SVD, compute the eigenvalues $\sigma_{i}$ of $y$.

- The geodesic distance is $d\left(p_{1}, p_{2}\right)=\left(\sum_{i=1}^{3} \log \left(\sigma_{i}\right)^{2}\right)^{\frac{1}{2}}$.

\subsection{Statistics of Diffusion Tensors}

We now define the mean and variance of diffusion tensors respecting the geometry of the space. Following Fréchet [9], we define the average as the minimum mean squared error estimator under the natural Riemannian metric defined above. Given a set of diffusion tensors $p_{1}, \ldots, p_{N} \in P D(3)$ the mean is defined as

$$
\mu=\underset{p \in P D(3)}{\arg \min } \sum_{i=1}^{N} d\left(p, p_{i}\right)^{2} .
$$

This minimization problem can be solved using a gradient descent method as described in [5]. This is analogous to the algorithm for computing the intrinsic mean given by Pennec [10]. Having defined the mean, we define the sample variance of the data as the expected value of the squared geodesic distances from the mean:

$$
\sigma^{2}=\frac{1}{N} \sum_{i=1}^{N} d\left(\mu, p_{i}\right)^{2}
$$

\subsection{Interpolation of Tensors}

For developing consistent interpolation between diffusion tensors we extend the above definition of the mean to weighted averaging. Using a least-squares criterion, we define the weighted average of diffusion tensors $p_{1}, \ldots, p_{N} \in P D(3)$ as

$$
\operatorname{Ave}\left(\left\{w_{i}\right\},\left\{p_{i}\right\}\right)=\underset{p \in P D(3)}{\arg \min } \sum_{i=1}^{N} w_{i} d\left(p, p_{i}\right)^{2},
$$
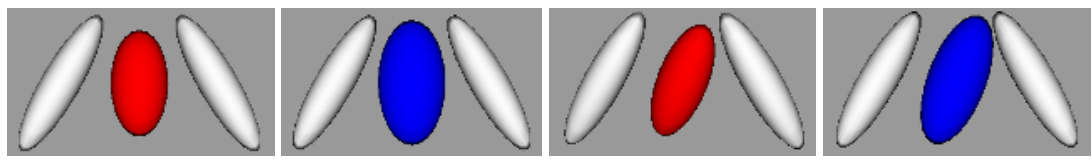

Fig. 1. Synthetic examples of weighted averages of tensors. The white ellipsoids average to the red ellipsoid with the geodesic method and to the blue ellipsoid with the linear method. Left: weights $=\{0.5,0.5\}$. Right: weights $=\{0.75,0.25\}$. It can be observed that the linear method does not preserve the determinant. 
where $w_{1}, \ldots, w_{N}$ are positive real weights that sum to 1 . Figure 1 provides synthetic examples of weighted averages of tensors.

For interpolating tensors within a voxel, trilinear weights may be used for the $w_{i}$. In this paper, we only focus on trilinear weights although higher order interpolation may be defined using the same concept. This interpolation is a natural generalization of trilinear interpolation of scalar values, i.e., if we replaced the diffusion tensors in the above definitions with real numbers, we would arrive at trilinear interpolation. It follows easily from the use of trilinear weights that the interpolation function does indeed interpolate the corner points. It can also be shown that the interpolation function is continuous on $[0,1]^{3}$ (see [6] for a proof).

\section{Fiber Tract Modeling and Analysis Methodology}

Interpolation and averaging of tensors is applied for quantitative fiber tract-oriented analysis of DTI. The geometry of an individual fiber tract is modeled, basically with what is commonly called a point distribution model (PDM) [11]. Diffusion tensor statistics are computed across fiber tract sections and are associated with the mean geometric model, resulting in a compact description of diffusion properties along the fiber tract. An overview of our framework is illustrated in Fig. 2 .

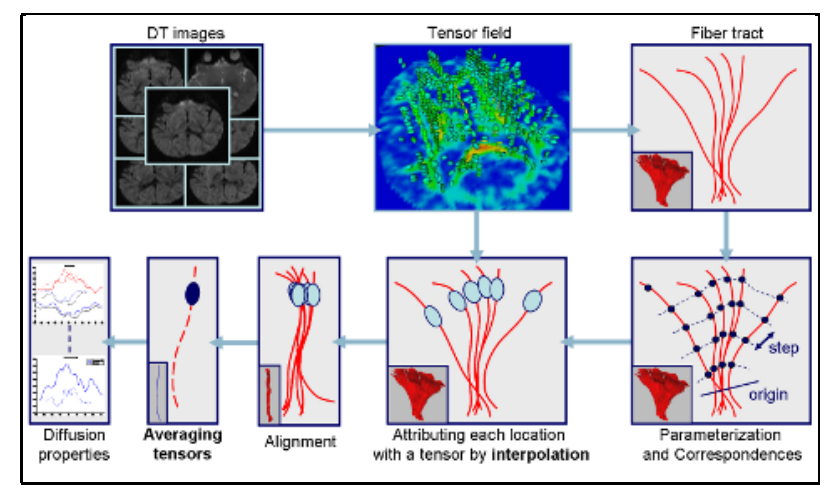

Fig. 2. Overview of the DTI analysis framework

\subsection{Preprocessing: Tensor Field Computation and Fiber Extraction}

The tensor field is computed from DTI data by solving the Stejskal-Tanner's diffusion equation system [12]. A tractography algorithm [13] extracts streamlines following the principal diffusion tensor directions between source and target regions of interest, with sub-voxel precision. Our latest version of the tractography tool includes tensor interpolation as described in Sect. 2.2. Except at branching or crossing points, the extracted 3D curves are assumed to represent the most likely pathways through the tensor field. 


\subsection{Geometric Modeling}

An individual fiber tract, described by a set of streamlines, acts as a training set from which we estimate a template shape, the mean shape, and statistical deviations by learning its inherent shape variability. A brief review of the geometric modeling of one individual fiber tract is presented below (see [4] for a more comprehensive description).

First, fibers represented as polylines are reparametrized by cubic B-spline curves. This ensures an equidistant sampling along each fiber as well as a consistent sampling for all fibers. Second, an origin, which can be reliably identified across subjects, is defined for each fiber tract. This is either a geometric criterion, e.g., a cross-section with minimal area, or anatomical information like intersection with the midsagittal plane. Points with the same arc-length along the fiber tract are defined as homologuous. This explicit point to point matching has been proven relevant in [2] where we demonstrated that it properly aligns local shape features across all curves in a fiber bundle. Given this correspondence, the alignment of all curves in the training set is achieved by Procrustes analysis [14]. Only estimated translations and rotations are applied to fibers. Indeed, a size normalization is not desirable since the training fibers belong to the same individual fiber tract. Given the set of aligned shapes, the mean shape is estimated by averaging the spatial coordinates at each corresponding location over the tract. Additionally, statistical shape deviations from this template shape along the tract can be characterized by extracting the principal modes of deformation via a principal component analysis.

\subsection{Attributing the Geometric Model with Diffusion Tensor Statistics}

The estimated mean shape models the geometry of the fiber tract. A complete representation of the tract, describing both geometry and diffusion properties, is obtained by attributing each location along the mean curve with statistics of diffusion tensors calculated over cross-sections.

Computing the Mean Tensors along the Fiber Tract. First, each sample point $x$ from the set of reparameterized fibers is assigned a tensor $p$. Since the tensor field is defined on the discrete voxel grid while $x$ lies on a continuous curve, a geodesic interpolation (see Sect. 2.2) is required to compute the tensor $p$ at the location $x$. The tensor $p$ is given by the weighted average of the eight voxel tensor values in the nearest $2 \times 2 \times 2$ neighborhood of $x$, the weights being defined by trilinear interpolation (see (3)). Let $\mathcal{P}$ be the set of obtained tensors, $\mathcal{P}=\left\{p_{f, i}\right\}$ with $f$ indexing the reparameterized fibers across the tract and $i$ the location along each reparameterized fiber. Then, the tensor set $\mathcal{P}$ is aligned by rotation. Let $R_{f} \in S O(3)$ be the rotation estimated by Procrustes analysis for the reparameterized fiber $f$. Each tensor $p_{f, i}$ lying on $f$ is rotated to the tensor $p_{f, i}^{\prime}$ by the group action $R_{f}: p_{f, i}^{\prime}=R_{f} p_{f, i} R_{f}^{T}, \forall i$. Last, at each corresponding location $i$ along the tract, the mean tensor $\mu_{i}$ is computed from the set of aligned tensors, $\left\{p_{f, i}^{\prime}\right\}$, as defined in (1). In addition, tensor diffusion variability can be assessed at each location of the average curve by computing the geodesic standard deviation according to (2). For visualization purposes, each average tensor is translated to its corresponding average location on the average curve. Since diffusion tensors are invariant to translation, this does not affect any diffusion property. 
Deriving Diffusion Properties. At each location along the template curve, diffusion properties are derived from the average tensor. We consider the measures: $i$ ) the three eigenvalues of the average diffusion tensor, $\lambda_{1}, \lambda_{2}, \lambda_{3}$, which represent the diffusivities along the three principal directions of the tensor, $i i)$ the mean diffusivity, MD, defined by the first moment of the diffusion tensor eigenvalues, iii) the fractional anisotropy, FA, which is a normalized measure of the tensor shape and defines a distance to isotropy.

\section{Experiments and Results}

We have applied our new fiber tract-oriented DTI analysis technique to image data of a prospective study of neonatal brain structure in children at high risk for schizophrenia [15]. This study includes 3-Tesla MRI and DTI of neonates at 2 weeks of age with follow-up at 1 year. Local diffusion properties in white matter as measured by DTI have been implicated to be associated with axon density, degree of myelination and density of fluid. DTI of neonates in general present a decrease of FA and an increase of MD from central to peripheral regions, reflecting the typical pattern of early structuring of white matter. Over the first few months till age 1 and further, there is a rapid development of myelination of white matter. This is expected to be demonstrated by a thinning of local tensors and therefore by an increase of FA and a decrease of MD as a function of age. We will focus on the commissural bundles of the corpus callosum, specifically on the genu and splenium fiber tracts which connect prefontal cortices and parts of the temporal, parietal and occipital lobes, respectively. It is known that the splenium is myelinating earlier than the genu, and that the presence of myelin sheaths has a significant effect on ability of water to diffuse [16].

Diffusion Tensor Image Data. Neonates and follow-ups were scanned on a Siemens head-only 3T scanner (Allegra). A single shot echo planar (EPI) diffusion tensor sequence with total scan time of approximately 4 minutes was used. The imaging parameters were: $\mathrm{TR} / \mathrm{TE} / \mathrm{TH}=4219 \mathrm{~ms} / 92.2 \mathrm{~ms}$, isotropic voxels with $2 \mathrm{~mm}$ slice distance and inplane resolution $=2 \times 2 \mathrm{~mm}, 5$ averages, and number of slices ranging from 44 to 65 . Seven images were acquired for each slice, one without diffusion gradient $(b=0)$ while the remaining six with $b=1000 \mathrm{~s} / \mathrm{mm}^{2}$ and diffusion gradients along the standard orientations [17]. We selected 8 cases from our large image database: 4 neonates at 2 weeks and 4 infants at age 1 year. For all cases, the genu and splenium tracts were extracted by tractography (see Fig. 3), the regions of interest being manually defined on the FA image using our SNAP tool.

Average of Diffusion Tensors in Cross-Sections Along Tracts. The geometric model and associated diffusion tensor statistics are computed for both tracts of each subject as described in Sect. 3. The Procrustes alignment for clustering of dispersed bundles has been skipped in these experiments, because tracking resulted in coherent, compact bundles. Figure 4 shows the mean tensors along the mean curve for each selected fiber tract for typical neonate and one year-old cases. As expected, the one year-old case presents much sharper and more elongated mean tensors than the 2 weeks-old case, which is explained by myelination of these white matter tracts. 

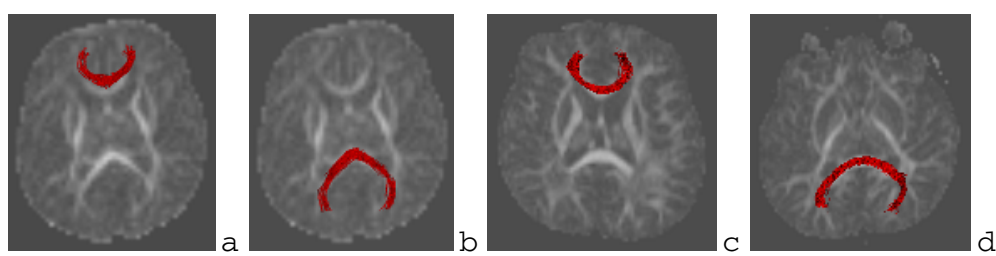

Fig. 3. DTI data with fiber tracts overlaid on axial sections of FA images. Genu and splenium of: a) , b) one typical neonate case, c), d) one typical one year-old case.
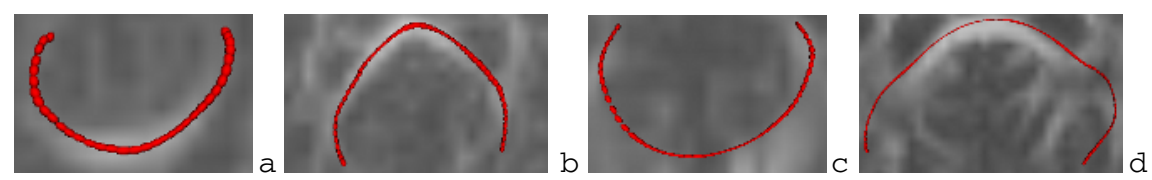

Fig. 4. Average tensors calculated in cross-sections displayed along central spine of each bundle. Genu and splenium of: a), b) one typical neonate case, c) , d) one typical one year-old case.

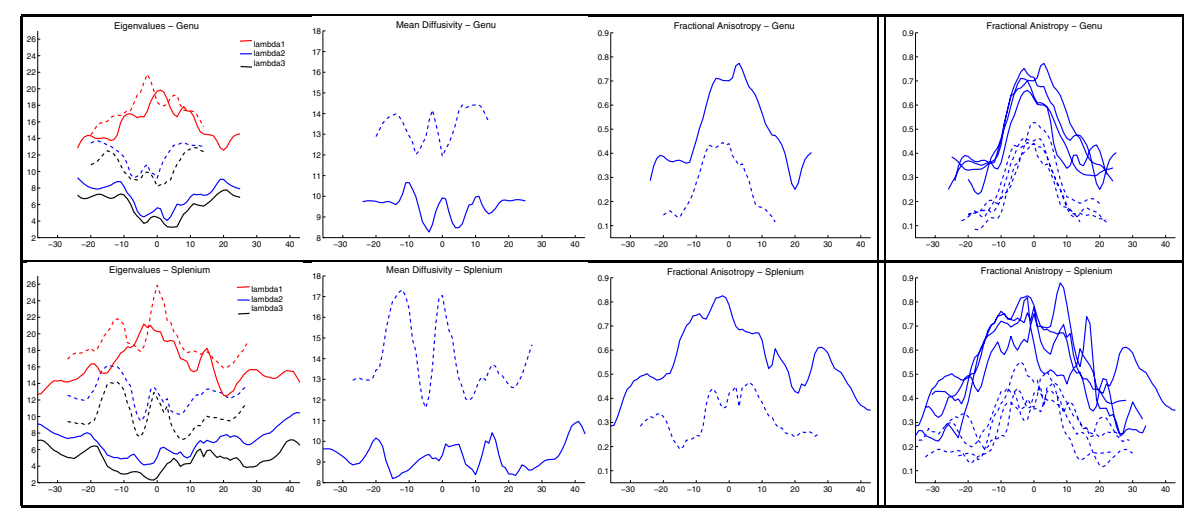

Fig. 5. Diffusion properties derived from average tensors plotted as a function of arc-length. From left to right: Eigenvalues, MD and FA for the genu (top row) and the splenium (bottom row) of one typical neonate (dashed lines) and one typical one year-old (solid lines). Last column displays FA for all 8 subjects: 4 neonates (dashed lines) and 4 one year-old (solid lines).

Diffusion Properties Along Fiber Tracts. The diffusion properties computed from the mean tensors, confirm the observation of increase of tensor elongation (see Fig. 5). For both tracts, the maximum diffusivity, $\lambda_{1}$, appears quite similar for all cases, whereas the median and mininum diffusivities, $\lambda_{2}$ and $\lambda_{3}$, are definitely higher in the two weeks-old cases (see dashed versus solid lines). Similarly, the mean diffusivity is much higher in the two weeks-old cases compared to the one year-old cases. This is consistent with the FA plots that, on the contrary, show higher values for the one year-old cases. These 
observed diffusion properties complies with the fact that white matter becomes more and more dense and structured with age. The overall pattern of each diffusion property for all subjects is similar. This indicates an early local structure of unmyelinated fiber tracts at birth, possibly explained by high axonal density, that develops into a more organized structure due to myelination with increasing age.

Validation. A preliminary validation of our quantitative DTI analysis framework has been performed on the basis of 6 repeated DTI scans of the same subject with slight change of head position [18]. The analysis is applied to 3 callosal bundles and the uncinate fasciculus from each single scan and an average DTI computed after rigid body alignment. Diffusion properties along tracts are compared between scans. Standard deviation from the set of 6 scans lies with $5 \%$ for FA and MD and 2\% for $\lambda_{1}$. This test/re-test validation demonstrated a good reproducibility of the methodology.

\section{Discussion}

We have presented a new framework for fiber tract-oriented quantitative analysis of DTI data. It combines a geometric model of fiber tracts with diffusion tensor statistics. Unlike most other statistical analysis of DTI data, we do not compute statistics on scalar measurements derived from tensors but we compute statistics on diffusion tensors followed by calculation of tensor properties. We use non linear statistics for tensor interpolation and averaging. The extended set of features provided by the new methodology which includes integrated measurements across bundles and along fiber tracts seems to be suitable to study white matter fiber tract properties in cross-sectional and longitudinal studies. The new quantitative DTI analysis technique describes properties of tracts and is therefore superior to conventional region-of-interest measurements and might lead to an improved understanding of MRI/DTI findings and its association to normal/abnormal brain development at early age. Our analysis confirms earlier findings in regard to decrease of FA and increase of MD towards peripheral regions. The expected change over the first year of development is clearly demonstrated by FA and MD plotted as a function of arc-length. Analysis of the three associated eigenvalues reveals more insight, namely that the FA increase is due to decrease of the second and third eigenvalue whereas the diffusion along the major tensor direction remains similar. Further analysis on a larger population with extended age range might reveal more insight into the trajectory of growth as measured by DTI and measured as a function of anatomical location. We presently have 60 neonates and will increase this number to a total of 125 . Ultimately, the proposed compact representation of the geometry of a tract and of associated diffusion properties aims at being used for inter-subject comparison and statistical analysis. This implies correspondence issues that are currently investigated by arc-length parameterization and could include local shape features of curves which have been shown to yield typical patterns along major fiber tracts [2]. Group comparison in clinical studies would require more advanced statistical techniques, for instance for comparison of probability distributions of tensors and hypothesis testing. 


\section{References}

1. Ding, Z., Gore, J., Anderson, A.: Classification and quantification of neuronal fiber pathways using diffusion tensor MRI. Magnetic Resonance in Medicine 49 (2003) 716-721

2. Corouge, I., Gouttard, S., Gerig, G.: Towards a shape model of white matter fiber bundles using Diffusion Tensor MRI. In: Proc. IEEE ISBI. (2004) 344-347

3. Lim, K., Helpern, J.: Neuropsychiatric applications of DTI - a review. NMR in Biomedicine 15 (2002) 587-593

4. Corouge, I., Gouttard, S., Gerig, G.: A statistical shape model of individual fiber tracts extracted from Diffusion Tensor MRI. In: MICCAI. Vol. 3217 of LNCS. (2004) 671-679

5. Fletcher, P.T., Joshi, S.: Principal geodesic analysis on symmetric spaces: Statistics of diffusion tensors. In: ECCV 2004, Workshop CVAMIA, Vol. 3117 of LNCS. (2004) 87-98

6. Fletcher, P.T.: Statistical variability in nonlinear spaces: Application to shape analysis and DT-MRI. PhD thesis, University of North Carolina (2004)

7. Batchelor, P.G., Moakher, M., Atkinson, D. Calamante, F., Connelly, A. A rigorous framework for diffusion tensor calculus. Magnetic Resonance in Medicine 53 (2005) 221-225

8. Helgason, S.: Differential Geometry, Lie Groups, and Symmetric Spaces. Academic Press (1978)

9. Fréchet, M.: Les éléments aléatoires de nature quelconque dans un espace distancié. Ann. Inst. H. Poincaré (1948) 215-310

10. Pennec, X.: Probabilities and statistics on Riemannian manifolds: basic tools for geometric measurements. In: IEEE Workshop on Nonlinear Signal and Image Processing. (1999)

11. Cootes, T., Taylor, C., Cooper, D., Graham, J.: Active shape models - their training and application. Computer Vision and Image Understanding 61 (1995) 38-59

12. Westin, C.F., Maier, S., Mamata, H., Nabavi, A., Jolesz, F., Kikinis, R.: Processing and visualization for diffusion tensor MRI. Medical Image Analysis 6 (2002) 93-108

13. Fillard, P., Gerig, G.: Analysis tool for diffusion tensor MRI. In: MICCAI. Vol. 2879 of LNCS. (2003) 967-968

14. Goodall, C.: Procrustes methods in the statistical analysis of shape. J.R. Statist. Soc. B 53 (1991) 285-239

15. Gilmore, J., Zhai, G., Wilber, K., Smith, J., Lin, W., Gerig, G.: 3T magnetic resonance imaging of the brain in newborns. Psychiatry Research Neuroimaging 132 (2004) 81-85

16. Rutherford, M.: MRI of the Neonatal Brain. WB Saunders Ltd. (2002) ISBN: 0702025348.

17. Basser, P., Pierpaoli, C.: Microstructural and physiological features of tissues elucidated by quantitative-diffusion-tensor MRI. J. Magn. Reson. B 3 (1996) 209-219

18. Gerig, G., Corouge, I., Vachet, C., Krishnan, K.R., MacFall, J.R.: Quantitative analysis of diffusion properties of white matter fiber tracts: A validation study. In: ISMRM, May 2005. 